\title{
Space-Time Coding for Single Carrier Block CDMA Systems
}

\author{
César A. Medina
}

Raimundo Sampaio-Neto

Tiago T. V. Vinhoza

\begin{abstract}
Resumo-Neste trabalho é proposto um esquema unificado para sistemas de transmissão CDMA por blocos em portadora única e codificação espaço-temporal. O sistema proposto utiliza as componentes das múltiplas antenas e dos múltiplos percursos do canal CDMA como fonte de informação redundante para atingir máximo ganho de diversidade, $G_{d}=n_{T} L$, onde $n_{T}$ é o número de antenas e $L$ é o número de percursos dos canal. O sistema proposto permite explorar a propriedade de ortogonalidade dos códigos de Alamouti e decodificar cada símbolo separadamente, reduzindo assim o custo computacional.
\end{abstract}

Palavras-Chave-Códigos espaço-temporais por blocos, máximo ganho de diversidade, sistemas de transmissão CDMA em portadora única.

Abstract-In this work we propose a unified framework for single carrier space-time CDMA block transmission systems with Alamouti's code. The proposed system uses the multiantenna and multipath components as redundant information to produce schemes with full diversity gain, $G_{d}=n_{T} L$, where $n_{T}$ is the number of antennas and $L$ is the number of multipath components in the transmission. The proposed system allow us to exploit the orthogonal property of Alamouti's code and to decode each transmit symbol separately, thus, reducing the computational complexity.

Keywords-Space-Time block codes, maximum diversity gain, single Carrier CDMA transmission systems.

\section{INTRODUCTION}

Differently from second generation $(2 \mathrm{G})$ systems, designed mainly for voice transmission, $3 \mathrm{G}$ must support variable data rates, depending on demand and level of mobility. Typically $144 \mathrm{~Kb} / \mathrm{s}$ is supported for full vehicular mobility and higher bandwidths for pedestrian levels of mobility [1]. Wideband CDMA like systems have been widely proposed to support this bandwidth-on-demand environment.

The use of the well known CDMA with a guard interval between symbols leads to two systems, namely, single carrier CDMA with cyclic prefix (SC CDMA CP) [2], [3] and single carrier CDMA with zero padding (SC CDMA ZP). In both cases, the chips are transmitted sequentially over the whole bandwidth allocated for that user.

For the case of Beyond $3 G$ systems these requirements are even stronger, with data rate transmission of $100 \mathrm{Mb} / \mathrm{s}$ for mobile and $1 \mathrm{~Gb} / \mathrm{s}$ for stationary users [1]. One key to achieve this performance is the use of multiple input, multiple output (MIMO) technology, which uses multiple antennas to transmit

César A. Medina, Raimundo Sampaio-Neto and Tiago T. V. Vinhoza are with the Centro de Estudos em Telecomunicações (CETUC), Pontifícia Universidade do Rio de Janeiro (PUC-Rio), Rio de Janeiro (RJ), Brazil, E-mails: \{csmedina, raimundo,vinhoza\}@ cetuc.puc-rio.br. This work was supported by the Brazilian Council for Scientific and Technological Development (CNPq). and receive signals. MIMO has gained a lot of attention as an effective diversity technique to combat fading and/or increase the capacity of wireless networks without increasing the bandwidth or the transmitted signal power [1], [4]. This robustness is achieved by the use of techniques such as the Alamouti space-time code [5], which uses two transmit and one or multiple receive antennas. Alamouti's code is the only existing complex orthogonal design with full rate, full diversity and minimal delay [4], [6].

In this work we propose a unified framework to single carrier space-time CDMA block systems with Alamouti's code. Two types of guard interval are considered: cyclic prefix and zero padding. The diversity gain is analyzed and the conditions to achieve this gain are obtained. We exploit the orthogonal property of Alamouti's code to decode each transmit symbol separately with the decision directed minimum mean squared error (DD-MMSE) receiver, although any other receiver can be used.

This paper is organized as follows: Section II describes a unified framework of a Alamouti block transmission system. Section III addresses the definitions and properties of the employed matrices for both SC CDMA systems. In Section IV the diversity gain analysis is performed and the conditions to achieved such diversity are analyzed. Section $\mathrm{V}$ presents the decision directed minimum mean squared error receiver to decode each symbols separately and in Section VI we present the results obtained trough computer simulations. Finally, Section VII gives the conclusions.

Notation: In what follows, $\boldsymbol{I}_{k}$ represents a $k \times k$ identity matrix, $\mathbf{0}_{m \times n}$, an $m \times n$ null matrix, $(\cdot)^{T},(\cdot)^{H}$ and $(\cdot)^{*}$ denote transpose, Hermitian transpose and complex conjugated, respectively, $\odot$ represents the pointwise product, $\operatorname{diag}(\boldsymbol{x})$ is a diagonal matrix with the components of $\boldsymbol{x}$ as its nonzero elements, $\otimes$ is the Kronecker product and the operator $\mathbb{E}[\cdot]$ stands for ensemble average.

\section{Space-Time Coding For Single CARrier Block TRANSMISSION SYSTEMS}

A discrete model of a downlink block space-time system employing Alamouti's [5] scheme is depicted in Fig. 1. This scheme uses two transmit antennas and one receive antenna. The system is described as follows. Each user transmits symbols $s_{k}(i)$ which are first spreaded by the spreading code $c_{k}$. It is assumed that symbols $s_{k}(i)$, drawn from some constellation with zero mean and unit average symbol energy, are independent and identically distributed (i.i.d.) and that the spreading code is composed by $M$ chips per symbol. 


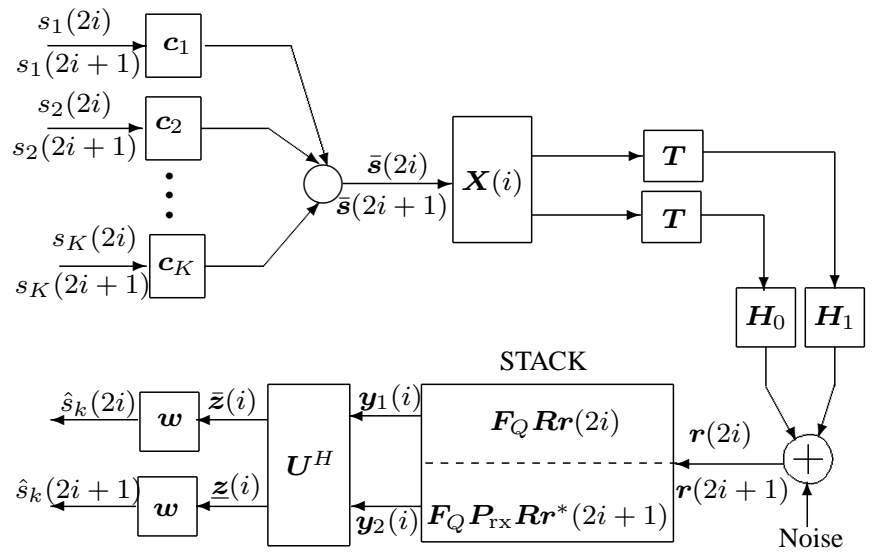

Fig. 1. Space-Time Coding for Single Carrier CDMA Block Transmission Systems

If we define $\boldsymbol{C}=\sqrt{\tilde{E}_{s}} \overline{\boldsymbol{C}} \boldsymbol{\rho}$, where $\tilde{E}_{s}=\left(E_{1}+E_{2}+\cdots+\right.$ $\left.E_{K}\right) / K$ is the mean transmitted energy, $E_{k}$ is the transmitted energy for user $k, \boldsymbol{\rho}=\operatorname{diag}\left(\rho_{1} \rho_{2} \cdots \rho_{K}\right), \rho_{k}=\sqrt{E_{k} / \tilde{E}_{s}}$ and $\overline{\boldsymbol{C}}=\left[\begin{array}{llll}\boldsymbol{c}_{1} & \boldsymbol{c}_{2} & \cdots & \boldsymbol{c}_{K}\end{array}\right]$, then the space-time coding matrix maps vectors $\overline{\boldsymbol{s}}(i)=\sum_{k=1}^{K} \sqrt{E_{k}} \boldsymbol{c}_{k} s_{k}(i)=\boldsymbol{C} \boldsymbol{s}(i)$, as

$$
\boldsymbol{X}(i)=\left(\begin{array}{cc}
\overline{\boldsymbol{s}}(2 i) & \boldsymbol{P}_{\mathrm{tx}} \overline{\boldsymbol{s}}^{*}(2 i+1) \\
\overline{\boldsymbol{s}}(2 i+1) & -\boldsymbol{P}_{\mathrm{tx}} \overline{\boldsymbol{s}}^{*}(2 i)
\end{array}\right)
$$

where $\boldsymbol{s}(i)=\left[\begin{array}{llll}s_{1}(i) & s_{2}(i) & \cdots & s_{K}(i)\end{array}\right]^{T}, \boldsymbol{P}_{\mathrm{tx}}$ is a $M \times M$ permutation matrix which depends of the system employed as detailed in the next section.

Before transmission a guard interval insertion is performed by the $P \times M$ matrix $\boldsymbol{T}$, where $P=M+G$ and $G$ is the length of the guard interval. For the most commonly used guard intervals, cyclic prefix (CP) and zero padding (ZP) [7], the matrix $\boldsymbol{T}$ is defined as:

$$
\boldsymbol{T}_{z p}=\left[\begin{array}{c}
\boldsymbol{I}_{M} \\
\mathbf{0}_{G \times M}
\end{array}\right] \quad \boldsymbol{T}_{c p}=\left[\begin{array}{c}
\mathbf{0}_{G \times(M-G)} \mid \boldsymbol{I}_{G} \\
\boldsymbol{I}_{M}
\end{array}\right]
$$

The channel impulse response from the $j$-th $(j=1,2)$ transmission antenna to the receiver is modeled here as a FIR filter with $L$ taps whose gains are samples of the channel impulse response complex envelope. Assuming that during two symbol periods each multipath channel impulse response remains constant, that is, $\boldsymbol{h}_{j}(2 i)=\boldsymbol{h}_{j}(2 i+1)=$ $\left[h_{j, 0}(2 i) \ldots h_{j, L-1}(2 i)\right]^{T}$, the transmission through the multipath channel can be represented by a $P \times P$ lower triangular Toeplitz convolution matrix $\boldsymbol{H}_{j}(2 i)$, whose first column is

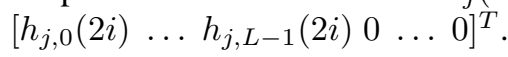

As we assume a downlink scenario, where users experience the same channel condition, the received signal vectors collected over two consecutive symbol periods are:

$$
\begin{aligned}
\boldsymbol{r}(2 i)= & \boldsymbol{H}_{0}(2 i) \boldsymbol{T} \overline{\boldsymbol{s}}(2 i) \\
& +\boldsymbol{H}_{1}(2 i) \boldsymbol{T} \overline{\boldsymbol{s}}(2 i+1)+\boldsymbol{n}(2 i)+\boldsymbol{\eta}(2 i) \\
\boldsymbol{r}(2 i+1)= & \boldsymbol{H}_{0}(2 i) \boldsymbol{T} \boldsymbol{P}_{\mathrm{tx}} \overline{\boldsymbol{s}}^{*}(2 i+1) \\
& -\boldsymbol{H}_{1}(2 i) \boldsymbol{T} \boldsymbol{P}_{\mathrm{tx}} \overline{\boldsymbol{s}}^{*}(2 i)+\boldsymbol{n}(2 i+1) \\
& +\boldsymbol{\eta}(2 i+1)
\end{aligned}
$$

where $\boldsymbol{n}(i)$ is a complex white Gaussian noise vector with zero mean and covariance matrix $\mathbb{E}\left[\boldsymbol{n}(i) \boldsymbol{n}^{H}(i)\right]=\frac{N_{0}}{2} \boldsymbol{I}_{P}$, $\boldsymbol{\eta}(i)$ represents the interblock interference (IBI).

For block transmission with $\mathrm{CP}$, the receiver must remove the guard interval from the received signal to eliminate IBI. In the ZP case, guard interval removal is not necessary. This operation is represented by the matrix $\boldsymbol{R}$, where $\boldsymbol{R}=\boldsymbol{R}_{z p}=$ $\boldsymbol{I}_{P}$ for ZP systems and $\boldsymbol{R}=\boldsymbol{R}_{c p}=\left[\mathbf{0}_{M \times G} \mid \boldsymbol{I}_{M}\right]$ for $\mathrm{CP}$ systems.

Finally, a space-time decoding is performed by stacking the signals as

$$
\boldsymbol{y}(i)=\left[\begin{array}{c}
\boldsymbol{y}_{1}(i) \\
\boldsymbol{y}_{2}(i)
\end{array}\right]=\left[\begin{array}{c}
\boldsymbol{F}_{Q} \boldsymbol{R} \boldsymbol{r}(2 i) \\
\boldsymbol{F}_{Q} \boldsymbol{P}_{\mathrm{rx}} \boldsymbol{R} \boldsymbol{r}^{*}(2 i+1)
\end{array}\right]
$$

where $Q=M$ for $\mathrm{CP}$ systems and $Q=P$ for $\mathrm{ZP}$ case, $\boldsymbol{P}_{\mathrm{rx}}$ is a $Q \times Q$ permutation matrix whose choice depends on the overall system employed as detailed in the next section and $\boldsymbol{F}_{Q}$ is a $Q \times Q$ matrix that implements a $Q$-point FFT, normalized such that, $\boldsymbol{F}_{Q}^{H} \boldsymbol{F}_{Q}=\boldsymbol{F}_{Q} \boldsymbol{F}_{Q}^{H}=\boldsymbol{I}_{Q}$.

\section{MATRiX Definitions}

The use of the appropriate matrices $\boldsymbol{T}$ and $\boldsymbol{R}$ as defined in the previous section, leads to two commonly used systems, namely, single carrier CDMA with cyclic prefix (SC CDMA $\mathrm{CP}$ ) and single carrier CDMA with zero padding (SC CDMA $\mathrm{ZP}$ ). These choices are interesting because they lead us use the following properties:

p1) CP case: $\boldsymbol{R}_{c p} \boldsymbol{H}_{j}(2 i) \boldsymbol{T}_{c p}$ reduces to a circulant matrix $\mathrm{H}_{j}(2 i)$ of dimension $M \times M$.

p2) ZP case: $\boldsymbol{R}_{z p} \boldsymbol{H}_{j}(2 i) \boldsymbol{T}_{z p}$ is equivalent to $\mathrm{H}_{j}(2 i) \boldsymbol{T}_{z p}$ where $\mathbb{H}_{j}(2 i)$ is a circulant matrix of dimension $P \times P$. The equivalence is due to the structure of $\boldsymbol{T}_{z p}$.

p3) A circulant matrix $\mathbb{H}_{j}(2 i)$, as in p1) and p2), can be decomposed as $\mathrm{H}_{j}(2 i)=\boldsymbol{F}_{Q}^{H} \boldsymbol{\Lambda}_{j}(2 i) \boldsymbol{F}_{Q}$ and $\mathrm{H}_{j}^{H}(2 i)=$ $\boldsymbol{F}_{Q}^{H} \boldsymbol{\Lambda}_{j}^{*}(2 i) \boldsymbol{F}_{Q}$, where $\boldsymbol{\Lambda}_{j}(2 i)$ is a diagonal matrix whose entries are the frequency response of the transmission channel $\boldsymbol{h}_{j}(2 i)$, i.e., $\boldsymbol{\Lambda}_{j}(2 i)=\operatorname{diag}\left(\tilde{\boldsymbol{F}} \boldsymbol{h}_{j}(2 i)\right)$, where $\tilde{\boldsymbol{F}}$ is a $Q \times L$ matrix formed with the first $L$ columns of the matrix that implements the (non-normalized) $Q$-point FFT.

\section{A. Permutation Matrices}

$\boldsymbol{P}_{\mathrm{tx}}$ and $\boldsymbol{P}_{\mathrm{rx}}$ are permutation matrices drawn from a set $\left\{\boldsymbol{P}_{J}^{(n)}\right\}_{n=0}^{J-1}$, where $J$ denotes dimension of $\boldsymbol{P}[8]$. Each $\boldsymbol{P}_{J}^{(n)}$ performs a reverse cyclic shift that depends on $n$ when applied to a $J \times 1$ vector.

These matrices have two properties which are shown bellow: p4) Pre- and postmultiplying a circulant matrix, $\mathrm{H}_{j}(2 i)$, by $\boldsymbol{P}_{J}^{(n)}$ yields $\mathbb{H}_{j}^{T}(2 i)$, i.e., $\boldsymbol{P}_{J}^{(n)} \mathbb{H}_{j}(2 i) \boldsymbol{P}_{J}^{(n)}=\mathbb{H}_{j}^{T}(2 i)$ and $\boldsymbol{P}_{J}^{(n)} \mathbb{H}_{j}^{*}(2 i) \boldsymbol{P}_{J}^{(n)}=\mathbb{H}_{j}^{H}(2 i)[8]$.

p5) $\boldsymbol{T}_{z p} \boldsymbol{P}_{M}^{(0)}=\boldsymbol{P}_{P}^{(M)} \boldsymbol{T}_{z p}[9]$.

The five properties above allow us to simplify the systems as follows. Using (2) we can rewrite (3) as

$$
\begin{aligned}
\boldsymbol{y}(i)= & {\left[\begin{array}{l}
\boldsymbol{f}_{0}\left(\boldsymbol{H}_{0}\right) \boldsymbol{s}(2 i)+\boldsymbol{f}_{0}\left(\boldsymbol{H}_{1}\right) \boldsymbol{s}(2 i+1) \\
\boldsymbol{f}_{1}\left(\boldsymbol{H}_{0}\right) \boldsymbol{s}(2 i+1)-\boldsymbol{f}_{1}\left(\boldsymbol{H}_{1}\right) \boldsymbol{s}(2 i)
\end{array}\right] } \\
+ & \underbrace{\left[\begin{array}{c}
\boldsymbol{F}_{Q} \boldsymbol{R} \boldsymbol{n}(2 i) \\
\boldsymbol{F}_{Q} \boldsymbol{P}_{\mathrm{rx}} \boldsymbol{R} \boldsymbol{n}^{*}(2 i+1)
\end{array}\right]}_{\overline{\boldsymbol{n}}(i)}
\end{aligned}
$$


where

$$
\begin{array}{r}
\boldsymbol{f}_{0}\left(\boldsymbol{H}_{j}\right)=\boldsymbol{F}_{Q} \boldsymbol{R} \boldsymbol{H}_{j}(2 i) \boldsymbol{T} \boldsymbol{C} \\
\boldsymbol{f}_{1}\left(\boldsymbol{H}_{j}\right)=\boldsymbol{F}_{Q} \boldsymbol{P}_{\mathrm{rx}} \boldsymbol{R} \boldsymbol{H}_{j}^{*}(2 i) \boldsymbol{T} \boldsymbol{P}_{\mathrm{tx}} \boldsymbol{C} .
\end{array}
$$

Note that the term $\boldsymbol{\eta}(i)$ in (2), which accounts for IBI, is removed by the joint operation of matrices $\boldsymbol{T}$ and $\boldsymbol{R}$. It should be stressed that the guard interval length must be at least the channel order in order to avoid IBI, i.e., $G \geq L-1$ [7].

1) SC CDMA CP: If we choose $\boldsymbol{P}_{\mathrm{rx}}=\boldsymbol{P}_{\mathrm{tx}}=\boldsymbol{P}_{M}^{(0)}$ and using $\mathrm{p} 1$ ), $\mathrm{p} 4$ ) and $\mathrm{p} 3$ ) we have:

$$
\begin{aligned}
& \boldsymbol{f}_{0}\left(\boldsymbol{H}_{j}\right)=\boldsymbol{\Lambda}_{j}(2 i) \boldsymbol{F}_{M} \boldsymbol{C}=\boldsymbol{\Lambda}_{j}(2 i) \boldsymbol{V} \\
& \boldsymbol{f}_{1}\left(\boldsymbol{H}_{j}\right)=\boldsymbol{\Lambda}_{j}^{*}(2 i) \boldsymbol{F}_{M} \boldsymbol{C}=\boldsymbol{\Lambda}_{j}^{*}(2 i) \boldsymbol{V}
\end{aligned}
$$

where $\boldsymbol{V}=\boldsymbol{F}_{M} \boldsymbol{C}=\sqrt{\tilde{E}_{s}} \overline{\boldsymbol{V}}$ and $\overline{\boldsymbol{V}}=\boldsymbol{F}_{M} \overline{\boldsymbol{C}} \boldsymbol{\rho}$.

2) SC CDMA ZP: We can choose $\boldsymbol{P}_{\mathrm{rx}}=\boldsymbol{P}_{P}^{(M)}$ and $\boldsymbol{P}_{\mathrm{tx}}=$ $\boldsymbol{P}_{M}^{(0)}$, and using p2) we have $\boldsymbol{f}_{0}\left(\boldsymbol{H}_{j}\right)=\boldsymbol{F}_{P} \mathrm{H}_{j}(2 i) \boldsymbol{T}_{z p} \boldsymbol{C}$ and $\boldsymbol{f}_{1}\left(\boldsymbol{H}_{j}\right)=\boldsymbol{F}_{P} \boldsymbol{P}_{P}^{(M)} \mathrm{H}_{j}^{*}(2 i) \boldsymbol{T}_{z p} \boldsymbol{P}_{M}^{(0)} \boldsymbol{C}$. Now, using p5), p4) and $\mathrm{p} 3$ )

$$
\begin{aligned}
& \boldsymbol{f}_{0}\left(\boldsymbol{H}_{j}\right)=\boldsymbol{\Lambda}_{j}(2 i) \boldsymbol{F}_{P} \boldsymbol{T}_{z p} \boldsymbol{C}=\boldsymbol{\Lambda}_{j}(2 i) \boldsymbol{V} \\
& \boldsymbol{f}_{1}\left(\boldsymbol{H}_{j}\right)=\boldsymbol{\Lambda}_{j}^{*}(2 i) \boldsymbol{F}_{P} \boldsymbol{T}_{z p} \boldsymbol{C}=\boldsymbol{\Lambda}_{j}^{*}(2 i) \boldsymbol{V}
\end{aligned}
$$

where $\boldsymbol{V}=\boldsymbol{F}_{P} \boldsymbol{T}_{z p} \boldsymbol{C}=\sqrt{\tilde{E}_{s}} \overline{\boldsymbol{V}}$ and $\overline{\boldsymbol{V}}=\boldsymbol{F}_{P} \boldsymbol{T}_{z p} \overline{\boldsymbol{C}} \boldsymbol{\rho}$.

Using the previous results, (4) can be expressed for the four models as

$$
\boldsymbol{y}(i)=\underbrace{\left[\begin{array}{cc}
\boldsymbol{\Lambda}_{0}(2 i) & \boldsymbol{\Lambda}_{1}(2 i) \\
-\boldsymbol{\Lambda}_{1}^{*}(2 i) & \boldsymbol{\Lambda}_{0}^{*}(2 i)
\end{array}\right]}_{\boldsymbol{\Lambda}(2 i)}\left[\begin{array}{c}
\boldsymbol{V} \boldsymbol{s}(2 i) \\
\boldsymbol{V} \boldsymbol{s}(2 i+1)
\end{array}\right]+\overline{\boldsymbol{n}}(i)
$$

where $\boldsymbol{V}$ is a matrix of dimension $Q \times K$ and is defined for each system as in before.

Defining $\boldsymbol{\Lambda}_{01}(2 i)=\left[\boldsymbol{\Lambda}_{0}^{*}(2 i) \boldsymbol{\Lambda}_{0}(2 i)+\boldsymbol{\Lambda}_{1}^{*}(2 i) \boldsymbol{\Lambda}_{1}(2 i)\right]^{1 / 2}$, then exists a unitary $2 Q \times 2 Q$ matrix $\boldsymbol{U}(2 i)=$ $\boldsymbol{\Lambda}(2 i)\left(\boldsymbol{I}_{2} \otimes \boldsymbol{\Lambda}_{01}^{-1}(2 i)\right)$, such that $\boldsymbol{U}^{H}(2 i) \boldsymbol{U}(2 i)=\boldsymbol{I}_{2 Q}$ and $\boldsymbol{U}^{H}(2 i) \boldsymbol{\Lambda}(2 i)=\boldsymbol{I}_{2} \otimes \boldsymbol{\Lambda}_{01}(2 i)$, then:

$$
\begin{aligned}
\boldsymbol{z}(i)= & \boldsymbol{U}^{H}(2 i) \boldsymbol{y}(i)=\left[\begin{array}{cc}
\boldsymbol{\Lambda}_{01}(2 i) & \mathbf{0}_{Q \times Q} \\
\mathbf{0}_{Q \times Q} & \boldsymbol{\Lambda}_{01}(2 i)
\end{array}\right] \times \\
& \times\left[\begin{array}{c}
\boldsymbol{V} \boldsymbol{s}(2 i) \\
\boldsymbol{V} \boldsymbol{s}(2 i+1)
\end{array}\right]+\boldsymbol{U}^{H}(2 i) \overline{\boldsymbol{n}}(i) .
\end{aligned}
$$

Then the blocks $\boldsymbol{s}(2 i)$ and $\boldsymbol{s}(2 i+1)$ can be demodulated separately

$$
\begin{array}{r}
\boldsymbol{z}_{0}(i)=\boldsymbol{\Lambda}_{01}(2 i) \boldsymbol{V} \boldsymbol{s}(2 i)+\overline{\boldsymbol{n}}_{0}(2 i) \\
\boldsymbol{z}_{1}(i)=\boldsymbol{\Lambda}_{01}(2 i) \boldsymbol{V} \boldsymbol{s}(2 i+1)+\overline{\boldsymbol{n}}_{1}(2 i+1)
\end{array}
$$

where $\boldsymbol{U}^{H}(2 i) \overline{\boldsymbol{n}}(i)=\left[\begin{array}{ll}\overline{\boldsymbol{n}}_{0}^{T}(2 i) & \overline{\boldsymbol{n}}_{1}^{T}(2 i+1)\end{array}\right]^{T} . \boldsymbol{U}^{H}(2 i)$ does not alter the statistical properties of the noise.

\section{Diversity Gain Analysis}

Let us drop the time index and use only one equation from (13). If $P\left(\varepsilon_{k} \mid \boldsymbol{h}_{0}, \boldsymbol{h}_{1}\right)$ is the conditional error probability of user $k$, then

$$
P\left(\varepsilon_{k} \mid \boldsymbol{h}_{0}, \boldsymbol{h}_{1}\right) \leq P_{B}\left(\varepsilon \mid \boldsymbol{h}_{0}, \boldsymbol{h}_{1}\right)
$$

where $P_{B}(\cdot)$ is the block (block of symbols of $K$ users) error probability and is given by

$$
P_{B}\left(\varepsilon \mid \boldsymbol{h}_{0}, \boldsymbol{h}_{1}\right)=\sum_{\boldsymbol{s} \in \chi} \sum_{\substack{\hat{\boldsymbol{s}} \in \chi \\ \hat{\boldsymbol{s}} \neq \boldsymbol{s}}} P\left(\hat{\boldsymbol{s}} \mid \boldsymbol{s}, \boldsymbol{h}_{0}, \boldsymbol{h}_{1}\right)
$$

where $\chi$ represents the set of possible values for $s$ and $P\left(\hat{\boldsymbol{s}} \mid \boldsymbol{s}, \boldsymbol{h}_{0}, \boldsymbol{h}_{1}\right)$ is the conditional probability of the event that the detected block is $\hat{s}$ when the transmitted block is $s(\hat{s} \neq s)$. For a minimum distance receiver, given $s$, the probability of selecting a given alternative $\hat{s}$ when all other alternatives are possible is upper bounded by the probability of a minimum distance receiver selecting $\hat{s} \neq s$ in a binary decision scenario. So, for a given $\boldsymbol{h}_{0}$ and $\boldsymbol{h}_{1}$,

$$
\begin{aligned}
P\left(\hat{\boldsymbol{s}} \mid \boldsymbol{s}, \boldsymbol{h}_{0}, \boldsymbol{h}_{1}\right) & \leq Q\left(\sqrt{\left\|\boldsymbol{\Lambda}_{01} \overline{\boldsymbol{V}}(\hat{\boldsymbol{s}}-\boldsymbol{s})\right\|^{2} \tilde{E}_{s} / 2 N_{0}}\right) \\
& \leq \exp \left\{-\left\|\boldsymbol{\Lambda}_{01} \overline{\boldsymbol{V}}(\hat{\boldsymbol{s}}-\boldsymbol{s})\right\|^{2} \gamma / 4\right\}
\end{aligned}
$$

where $\gamma=\tilde{E}_{s} / N_{0}$.

From (14)-(16), we have, for equiprobable symbols

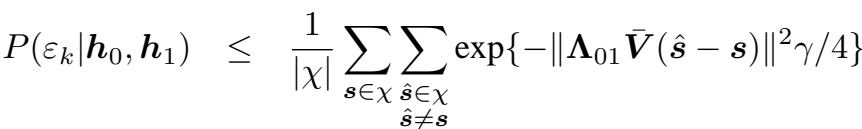

$$
\begin{aligned}
& \leq \frac{1}{|\chi|} \sum_{\boldsymbol{e} \neq \mathbf{0}} \vartheta(\boldsymbol{e}) \exp \left\{-\left\|\boldsymbol{\Lambda}_{01} \overline{\boldsymbol{V}} \boldsymbol{e}\right\|^{2} \gamma / 4\right\}
\end{aligned}
$$

where $|\chi|$ denotes the cardinality of $\chi, \boldsymbol{e}=\hat{\boldsymbol{s}}-\boldsymbol{s}$ and $\vartheta(\boldsymbol{e})$ is the number of occurrences of a given vector $e$. We then have,

$$
\begin{aligned}
P\left(\varepsilon_{k}\right) & \leq \frac{1}{|\chi|} \sum_{\boldsymbol{e} \neq \mathbf{0}} \vartheta(\boldsymbol{e}) \mathbb{E}_{\boldsymbol{h}_{0}, \boldsymbol{h}_{1}}\left[\exp \left\{-\left\|\boldsymbol{\Lambda}_{01} \overline{\boldsymbol{V}} \boldsymbol{e}\right\|^{2} \gamma / 4\right\}\right] \\
& \leq \frac{1}{|\chi|} \sum_{\boldsymbol{e} \neq \mathbf{0}} \vartheta(\boldsymbol{e}) f(\boldsymbol{e})
\end{aligned}
$$

where $\mathbb{E}_{\boldsymbol{h}_{0}, \boldsymbol{h}_{1}}[\cdot]$ stands for the average with respect to $\boldsymbol{h}_{0}$ and $\boldsymbol{h}_{1}$. To compute $f(\boldsymbol{e})$ we first proceed to rewrite $\left\|\boldsymbol{\Lambda}_{01} \overline{\boldsymbol{V}} \boldsymbol{e}\right\|^{2}$ as:

$$
\begin{aligned}
\left\|\boldsymbol{\Lambda}_{01} \overline{\boldsymbol{V}} \boldsymbol{e}\right\|^{2} & =\boldsymbol{e}^{H} \overline{\boldsymbol{V}}^{H}\left[\boldsymbol{\Lambda}_{0}^{*} \boldsymbol{\Lambda}_{0}+\boldsymbol{\Lambda}_{1}^{*} \boldsymbol{\Lambda}_{1}\right] \overline{\boldsymbol{V}} \boldsymbol{e} \\
& =\sum_{j=0}^{1}\left\|\boldsymbol{\Lambda}_{j} \boldsymbol{\beta}(\boldsymbol{e})\right\|^{2}=\sum_{j=0}^{1} \sum_{q=1}^{Q}\left|\beta_{q}(\boldsymbol{e}) \Lambda_{j, q}\right|^{2} \\
& =\sum_{j=0}^{1} \sum_{q=1}^{Q}\left|\beta_{q}(\boldsymbol{e}) \tilde{\boldsymbol{f}}_{q}^{H} \boldsymbol{h}_{j}\right|^{2}
\end{aligned}
$$

where $\beta_{q}(\boldsymbol{e})$ is the $q$-th element of $\boldsymbol{\beta}(\boldsymbol{e})=\overline{\boldsymbol{V}} \boldsymbol{e}, \Lambda_{j, q}$ is the $q$-th element of $\boldsymbol{\Lambda}_{j}$, and to arrive to the last expression we use p3), $\tilde{\boldsymbol{f}}_{q}^{H}$ denotes the $q$-th row of $\tilde{\boldsymbol{F}}$.

Let us denote $\boldsymbol{K}=\mathbb{E}\left[\boldsymbol{h}_{j} \boldsymbol{h}_{j}^{H}\right], j=0,1$, the covariance matrix of the channel vector $\boldsymbol{h}_{j}, j=0,1$, where it was assumed that the channels $\boldsymbol{h}_{j}$ are identically distributed. Since $\boldsymbol{K}$ is square Hermitian, it always admits spectral decomposition, i.e., $\boldsymbol{K}=\boldsymbol{\Omega} \boldsymbol{D} \boldsymbol{\Omega}^{H}$, where $\boldsymbol{D}$ is a $L \times L$ diagonal matrix whose entries are the eigenvalues of $\boldsymbol{K}$ and $\boldsymbol{\Omega}$ is a unitary matrix whose columns are the eigenvectors of $\boldsymbol{K}$.

If we introduce the channel vector $\tilde{\boldsymbol{h}}_{j}=\boldsymbol{D}^{-1 / 2} \boldsymbol{\Omega}^{H} \boldsymbol{h}_{j}$, which by construction has a identity covariance matrix, and 
define

$$
\boldsymbol{\Gamma}(\boldsymbol{e})=\sum_{q=1}^{Q}\left|\beta_{q}(\boldsymbol{e})\right|^{2}\left[\boldsymbol{D}^{1 / 2}\right]^{H} \boldsymbol{\Omega}^{H} \tilde{\boldsymbol{f}}_{q} \tilde{\boldsymbol{f}}_{q}^{H} \boldsymbol{\Omega} \boldsymbol{D}^{1 / 2}
$$

then (19) can be rewritten as

$$
\left\|\boldsymbol{\Lambda}_{01} \overline{\boldsymbol{V}} \boldsymbol{e}\right\|^{2}=\sum_{j=0}^{1} \tilde{\boldsymbol{h}}_{j}^{H} \boldsymbol{\Gamma}(\boldsymbol{e}) \tilde{\boldsymbol{h}}_{j}
$$

If the channels $\boldsymbol{h}_{j}$ are statistically independent, then based on (21) and averaging (16) with respect to $\boldsymbol{h}_{j}, j=0,1$, we get $f(\boldsymbol{e})$, for high signal to noise ratio, as [4]:

$$
f(\boldsymbol{e})=\left(\prod_{l=0}^{L} \frac{1}{\left(1+\gamma \lambda_{l}(\boldsymbol{e}) / 4\right)}\right)^{2}
$$

where $\lambda_{l}(\boldsymbol{e})$ are the eigenvalues of $\boldsymbol{\Gamma}(\boldsymbol{e})$. If $\kappa(\boldsymbol{e})$ is the rank of $\boldsymbol{\Gamma}(\boldsymbol{e})$, then $\lambda_{l}(\boldsymbol{e}) \neq 0$ if and only if $l \in[0, \kappa(\boldsymbol{e})-1]$, it thus follows from (22) that

$$
f(\boldsymbol{e}) \leq\left(\frac{\gamma}{4}\right)^{-2 \kappa(\boldsymbol{e})}\left(\prod_{l=0}^{\kappa(\boldsymbol{e})-1} \lambda_{l}(\boldsymbol{e})\right)^{-2}
$$

The value $G_{d}=2 \min _{\boldsymbol{e} \neq \mathbf{0}} \kappa(\boldsymbol{e})$ is called diversity gain [6], [10]. As the maximum $\operatorname{rank}$ of $\boldsymbol{\Gamma}(\boldsymbol{e})$ is $L$ (its dimension is $L \times$ $L)$, then we say that the system achieves maximum diversity gain if $\min _{\boldsymbol{e} \neq \mathbf{0}} \kappa(\boldsymbol{e})=L$, and thus, $\kappa(\boldsymbol{e})=L, \forall \boldsymbol{e} \neq \mathbf{0}$, resulting

$$
\begin{aligned}
P\left(\varepsilon_{k}\right) & \leq \frac{1}{|\chi|}\left(\frac{\gamma}{4}\right)^{-2 L} \sum_{\boldsymbol{e} \neq \mathbf{0}} \vartheta(\boldsymbol{e})\left(\prod_{l=0}^{L-1} \lambda_{l}(\boldsymbol{e})\right)^{-2} \\
& \leq \frac{1}{|\chi|}\left(\frac{\gamma}{4}\right)^{-2 L} \sum_{\boldsymbol{e} \neq \mathbf{0}} \frac{\vartheta(\boldsymbol{e})}{\operatorname{det}^{2}(\boldsymbol{\Gamma}(\boldsymbol{e}))}
\end{aligned}
$$

where $\operatorname{det}(\cdot)$ denotes determinant. Then, from (20) we can state the following sufficient (but not necessary) conditions to achieve maximum diversity gain.

Condition 4.1: Let $\Theta$ be the $L \times Q$ matrix whose columns are the $Q$ vectors $\boldsymbol{\theta}_{q}$ of dimension $L$, where $\boldsymbol{\theta}_{q}=$ $\left[\boldsymbol{D}^{1 / 2}\right]^{H} \boldsymbol{\Omega}^{H} \tilde{\boldsymbol{f}}_{q}$. If the matrix $\operatorname{diag}(\boldsymbol{\beta}(\boldsymbol{e})) \boldsymbol{\Theta}^{H}$ is full column rank, then maximum diversity gain is achieved by the system.

Proof: It is easy to show that with the above definitions of $\boldsymbol{\theta}_{q}$ and $\boldsymbol{\Theta}$, we can rewrite (20) as

$$
\boldsymbol{\Gamma}(\boldsymbol{e})=\sum_{q=1}^{Q}\left|\boldsymbol{\beta}_{q}(\boldsymbol{e})\right|^{2} \boldsymbol{\theta}_{q} \boldsymbol{\theta}_{q}^{H}=\boldsymbol{\Theta} \operatorname{diag}\left(\boldsymbol{\beta}^{*}(\boldsymbol{e}) \odot \boldsymbol{\beta}(\boldsymbol{e})\right) \boldsymbol{\Theta}^{H}
$$

then, from basic linear algebra, if $\operatorname{diag}(\boldsymbol{\beta}(\boldsymbol{e})) \Theta^{H}$ is full column rank, then $\boldsymbol{\Theta} \operatorname{diag}\left(\boldsymbol{\beta}^{*}(\boldsymbol{e}) \odot \boldsymbol{\beta}(\boldsymbol{e})\right) \boldsymbol{\Theta}^{H}$ is full rank, $\kappa(\boldsymbol{e})=L$, and the diversity gain is $G_{d}=2 L$.

Condition 4.2: In order to achieve the maximum diversity gain, $G_{d}=2 L$, is sufficient to guarantee that, for any $\boldsymbol{e} \neq \mathbf{0}$, at least $L$ elements from $\overline{\boldsymbol{V}} \boldsymbol{e}$ are different from zero.
Proof: First observe that by construction any $L$ columns of $\Theta$ are linearly independent. In fact, any $L$ vectors $\tilde{\boldsymbol{f}}_{q}$ are linearly independent (as they are rows of a matrix that implements a $Q$-point FFT of a $L$-point sequence), then, if we choose any $L$ vectors $\tilde{\boldsymbol{f}}_{q}$ of $\boldsymbol{\Theta}$ to form a full rank matrix $\boldsymbol{\Theta}_{L}$ and then pre-multiplying by the full rank matrix $\left[\boldsymbol{D}^{1 / 2}\right]^{H} \boldsymbol{\Omega}^{H}$, we get a full rank matrix, $\left[\boldsymbol{D}^{1 / 2}\right]^{H} \boldsymbol{\Omega}^{H} \boldsymbol{\Theta}_{L}$. This means that any $L$ vectors $\boldsymbol{\theta}_{q}=\left[\boldsymbol{D}^{1 / 2}\right]^{H} \boldsymbol{\Omega}^{H} \tilde{\boldsymbol{f}}_{q}$ of $\boldsymbol{\Theta}$ are linearly independent. Now, if $\overline{\boldsymbol{V}} \boldsymbol{e}$ has at least $L$ nonzero entries, then $\operatorname{diag}(\boldsymbol{\beta}(\boldsymbol{e}))=\operatorname{diag}(\overline{\boldsymbol{V}} \boldsymbol{e})$ has at least $L$ nonzero entries on its diagonal. Finally, we conclude that $\operatorname{diag}(\boldsymbol{\beta}(\boldsymbol{e})) \boldsymbol{\Theta}^{H}$ has at least $L$ nonzero rows, which by the preceding observation are linearly independent, then $\operatorname{rank}\left(\operatorname{diag}(\boldsymbol{\beta}(\boldsymbol{e})) \boldsymbol{\Theta}^{H}\right)=L$, which by Condition 4.1 guarantee maximum diversity.

\section{A. Maximum Diversity in the Proposed Systems}

It is well known that the use of maximum likelihood detection is necessary to exploit the full diversity gain [4], i.e., multichannel and multipath diversity. However, the choice of appropriate user codes allows suboptimum receivers to employ some of the multipath redundant information, as shown in the next sections.

1) $S C C D M A C P$ : In this system $\overline{\boldsymbol{V}}=\boldsymbol{F}_{M} \overline{\boldsymbol{C}} \boldsymbol{\rho}$. Then the user codes have to be carefully chosen in order to guarantee Condition 4.2 . One possibility is to choose $\bar{C}$ as:

$$
\overline{\boldsymbol{C}}=\frac{1}{\sqrt{M}} \boldsymbol{F}_{M}^{H}\left[\begin{array}{cccc}
1 & 1 & \cdots & 1 \\
\epsilon_{1} & \epsilon_{2} & \cdots & \epsilon_{K} \\
\vdots & \vdots & \ddots & \vdots \\
\epsilon_{1}^{M-1} & \epsilon_{2}^{M-1} & \cdots & \epsilon_{K}^{M-1}
\end{array}\right]
$$

where $\frac{1}{\sqrt{M}}$ normalizes the user codes, such that $\boldsymbol{c}_{k}^{H} \boldsymbol{c}_{k}=1$. The parameters $\epsilon_{k}$ can be chosen as equispaced points on the unit circle by setting $\epsilon_{k}=e^{-\sqrt{-1}(2 \pi / M) k}[11]$.

Note that this choice forces a Vandermonde structure in $\overline{\boldsymbol{V}}$, which guarantees that, for any $K$ dimensional vector $\boldsymbol{e} \neq \mathbf{0}$, $\overline{\boldsymbol{V}} \boldsymbol{e}$ has at most $K-1$ zeroes, or equivalently, at least $M-$ $K+1$ elements different form zero, then, to satisfy Condition 4.2, $M-K+1 \geq L$, or equivalently $K \leq M-L+1$.

Remark: Consider the use of Hadamard codes. Suppose only one user using the first code, $\boldsymbol{c}_{1}=(1 / \sqrt{M})[1, \cdots 1]^{T}$, then $\overline{\boldsymbol{V}}=\boldsymbol{F}_{M} \boldsymbol{c}_{1}=(1 / \sqrt{M})[1,0, \cdots 0]^{T}$. Note that a zero in the first subcarrier of $\boldsymbol{\Lambda}_{01}$ is sufficient to lose the diversity gain.

Furthermore, Condition 4.1 implies that $\Gamma(e)$ must be full rank, then (21) must be greater than zero for any channel vector in order to achieve maximum diversity gain. From (19) is possible to observe that for the distance to be equal to zero, the null elements of $\boldsymbol{\Lambda}_{01}$ must be located on the same subcarriers that the non null elements of $\boldsymbol{\beta}(\boldsymbol{e})$.

Nevertheless, is very unlikely that $\boldsymbol{\Lambda}_{0}$ and $\boldsymbol{\Lambda}_{1}$ have zeros over the same subcarriers at the same time, to produce a zero in $\boldsymbol{\Lambda}_{01}$ and even on the same subcarrier than a nonzero on $\boldsymbol{\beta}(\boldsymbol{e})$. Then, relaxing the conditions, we can conclude that other user codes than (26) can also achieve maximum diversity gain. 
2) SC CDMA ZP: In this system, $\overline{\boldsymbol{V}}=\boldsymbol{F}_{P} \boldsymbol{T}_{z p} \overline{\boldsymbol{C}} \boldsymbol{\rho}$. Lets denote $\overline{\boldsymbol{V}} \boldsymbol{e}=\tilde{\boldsymbol{F}}_{P \times M} \boldsymbol{e}^{\prime}$, where $\tilde{\boldsymbol{F}}_{P \times M}=\boldsymbol{F}_{P} \boldsymbol{T}_{z p}$ is a $P \times M$ matrix formed with the first $M$ columns of $\boldsymbol{F}_{P}$, and $\boldsymbol{e}^{\prime}=\overline{\boldsymbol{C}} \boldsymbol{\rho} \boldsymbol{e}$ is a $M$-dimensional vector. As $\bar{C}$ is full column rank, $e^{\prime} \neq \mathbf{0}$ when $\boldsymbol{e} \neq \mathbf{0}$.

If we note that the $P$-points discrete Fourier transform of a sequence of $M$ points can have at most $M-1$ zeros (because the Vandermonde structure of the matrix that implements the discrete Fourier transform), then $\tilde{\boldsymbol{F}}_{P \times M} \boldsymbol{e}^{\prime}$ has at least $P-$ $(M-1)=L+1$ elements different from zero, which by Condition 4.2 is sufficient to achieve maximum diversity gain. Note that maximum diversity gain is achieved independently from the choice of the user codes and the number of users on the system, provided that $\bar{C}$ is full column rank (the user codes are linearly independent).

\section{Decision Directed Minimum Mean Squared ERROR RECEIVER}

From (13) we can use the following equivalent expressions to derive the MMSE receiver:

$$
\boldsymbol{z}_{j}(i)=\boldsymbol{\Lambda}_{01}(2 i) \sum_{k=1}^{K} \boldsymbol{V}_{k} s_{k}(2 i+j)+\overline{\boldsymbol{n}}(2 i+j)
$$

where $\boldsymbol{V}_{k}$ is the $k$-th column of $\boldsymbol{V}$ and $j \in\{0,1\}$.

The $k$-th user MMSE receiver for both symbols, $\boldsymbol{w}_{k, M M S E}=\left[\begin{array}{ll}\boldsymbol{w}_{0, k, M M S E} & \boldsymbol{w}_{1, k, M M S E}\end{array}\right]$, is obtained by minimizing the mean-squared error criterion, whose solution is given by [12]:

$$
\boldsymbol{w}_{j, k, M M S E}=\boldsymbol{R}_{\boldsymbol{z}_{j} \boldsymbol{z}_{j}}^{-1} \boldsymbol{R}_{\boldsymbol{z}_{j} s_{k}}
$$

where $\boldsymbol{R}_{\boldsymbol{z}_{j} \boldsymbol{z}_{j}}=\mathbb{E}\left[\boldsymbol{z}_{j}(i) \boldsymbol{z}_{j}^{H}(i)\right], \boldsymbol{R}_{\boldsymbol{z}_{j} s_{k}}=\mathbb{E}\left[\boldsymbol{z}_{j}(i) s_{k}^{*}(2 i+j)\right]$.

The matrix $\boldsymbol{R}_{\boldsymbol{z}_{j} \boldsymbol{z}_{j}}^{-1}$ can be estimated recursively from the observation vector first using Kalman RLS recursions

$$
\hat{\boldsymbol{R}}_{\boldsymbol{z}_{j} \boldsymbol{z}_{j}}^{-1}(i)=\frac{1}{\alpha}\left[\hat{\boldsymbol{R}}_{\boldsymbol{z}_{j} \boldsymbol{z}_{j}}^{-1}(i-1)-\varphi_{j}(i) \boldsymbol{\Psi}_{j}(i) \boldsymbol{\Psi}_{j}^{H}(i)\right]
$$

where $0<\alpha<1$ is the forgetting factor, $\boldsymbol{\Psi}_{j}(i)$ is defined as the $Q$-dimensional Kalman gain vector and computed as

$$
\boldsymbol{\Psi}_{j}(i)=\hat{\boldsymbol{R}}_{\boldsymbol{z}_{j} \boldsymbol{z}_{j}}^{-1}(i-1) \boldsymbol{z}_{j}(i)
$$

and $\varphi_{j}(i)=\left[\frac{\alpha}{1-\alpha}+\boldsymbol{z}_{j}^{H}(i) \hat{\boldsymbol{R}}_{\boldsymbol{z}_{j} \boldsymbol{z}_{j}}^{-1}(i-1) \boldsymbol{z}_{j}(i)\right]^{-1}$.

The value for the input signal autocorrelation matrix at time zero is [13]

$$
\hat{\boldsymbol{R}}_{\boldsymbol{z}_{j} \boldsymbol{z}_{j}}(0)=E_{0} \operatorname{diag}\left(1, \alpha^{-1}, \alpha^{-2}, \cdots, \alpha^{-(Q-1)}\right)
$$

where $E_{0}$ is the forward prediction error energy and must be a positive value [13].

In order to compute $\boldsymbol{R}_{\boldsymbol{z}_{j} s_{k}}(i)$ recursively, a decision directed method can be employed. The complete algorithm is summarized next:

In the training phase, for $j \in\{0,1\}$ :

1) Compute $\hat{\boldsymbol{R}}_{\boldsymbol{z}_{j} \boldsymbol{z}_{j}}^{-1}(i)$, through (29);

2) compute $\hat{\boldsymbol{R}}_{\boldsymbol{z}_{j} s_{k}}(i)$ through

$$
\hat{\boldsymbol{R}}_{\boldsymbol{z}_{j} s_{k}}(i)=\alpha \hat{\boldsymbol{R}}_{\boldsymbol{z}_{j} s_{k}}(i-1)+(1-\alpha) \boldsymbol{z}_{j}(i) s_{k}^{*}(2 i+j) ;
$$

3) compute $\boldsymbol{w}_{j, k}(i)=\hat{\boldsymbol{R}}_{\boldsymbol{z}_{j} \boldsymbol{z}_{j}}^{-1}(i) \hat{\boldsymbol{R}}_{\boldsymbol{z}_{j} s_{k}}(i)$.
In the decision directed phase, for $j \in\{0,1\}$ :

1) Detection: $\hat{s}_{k}(2 i+j)=\operatorname{disc}\left\{\boldsymbol{w}_{j}^{H}(i-1) \boldsymbol{z}_{j}(i)\right\}$, where $\operatorname{disc}\{n\}$ is the symbol of the signal constellation closer to $n$;

2) compute $\hat{\boldsymbol{R}}_{\boldsymbol{z}_{j} \boldsymbol{z}_{j}}^{-1}(i)$ through (29);

3) compute $\hat{\boldsymbol{R}}_{\boldsymbol{z}_{j} s_{k}}(i)$ through

$$
\hat{\boldsymbol{R}}_{\boldsymbol{z}_{j} s_{k}}(i)=\alpha \hat{\boldsymbol{R}}_{\boldsymbol{z}_{j} s_{k}}(i-1)+(1-\alpha) \boldsymbol{z}_{j}(i) \hat{s}_{k}^{*}(2 i+j) ;
$$

4) compute $\boldsymbol{w}_{j, k}(i)=\hat{\boldsymbol{R}}_{\boldsymbol{z}_{j} \boldsymbol{z}_{j}}^{-1}(i) \hat{\boldsymbol{R}}_{\boldsymbol{z}_{j} s_{k}}(i)$.

\section{NumERicAl RESUltS}

In this section we evaluate the bit error rate (BER) performance of the proposed system based on the proposed decision directed minimum mean squared error receiver. We consider a BPSK signal constellation and four types of user codes: WalshHadamard, Vandermonde (in SC CDMA CP we force $\overline{\boldsymbol{V}}$ to be Vandermonde) and Zadoff-Chu [14] [15] codes, each of length 16 and pseudo-noise (PN) sequences of length 15 . We consider a scenario where the system has 4 users. Regarding power distribution, we simulate a severe near-far scenario where each interferer has a power level $20 \mathrm{~dB}$ above the desired user, that is, near-far ratio $(N F R)$ is equal to $20 \mathrm{~dB}$. For the timevariant channel, the sequence of channel coefficients for each user, $h_{l}(i)=p_{l} \alpha_{l}(i)(l=0,1,2, \ldots, L-1)$ is obtained with Clarke's model [16]. This procedure corresponds to the generation of independent sequences of correlated unit power complex Gaussian random variables $\left(\mathbb{E}\left[\left|\alpha_{l}^{2}(i)\right|\right]=1\right)$ with the path weights $p_{l}$ normalized so that $\sum_{l=0}^{L-1}\left|p_{l}\right|^{2}=1$. In this work $p_{l}=\sqrt{1 / L}, l=0,1,2, \ldots, L-1$, and a $f_{d} T=0.001$ was assumed. We simulate channels with length $L=4$ and $L=8$, and use a guard interval length equal to the channel order, i.e., $G=L-1$. The results are an average of 1000 experiments, and on each ensemble new codes are assigned to each user.

We consider perfect channel state information (CSI) at the receiver. In practical implementations, the CSI is not known at the receiver, but can be estimated by the use of pilot symbols.

In Fig. 2 and Fig. 3 we plot the BER versus the $E_{b} / N_{0}$ ( $E_{b}$ is the energy per bit of the desired user) for systems using the four types of codes. As can be observed the DDMMSE receiver can exploit, in almost all the cases, the spacetime diversity provided by the channel. With Hadamard codes, the SC CDMA CP system can not exploit the multipath components as predicted in Section IV. As verified in our studies, the use of Vandermonde codes in the SC CDMA ZP systems allows to exploit the multipath as well as the multichannel diversity if maximum likelihood detection is used. These results are not included here due to lack of space. The suboptimum receiver, however, cannot collect multipath diversity for this type of code.

In Fig. 4 the BER versus the $E_{b} / N_{0}$ for systems using different codes and the DD-MMSE receiver is shown. In order to compare with previously proposed systems, it is shown the performance of the system proposed in [17] and generalized to block CDMA systems in [18], which uses Walsh-Hadamard user codes and a receiver of the same type. A channel with $L=4$ paths was considered. It can be observed that the 

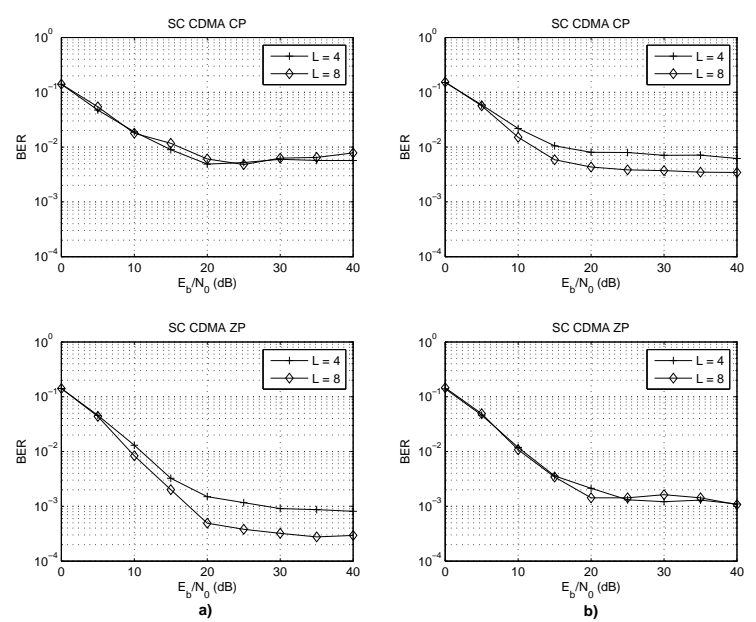

Fig. 2. BER versus $E_{b} / N_{0}$ for BPSK constellation with different channel orders, DD-MMSE receiver. a) Hadamard b) Vandermonde
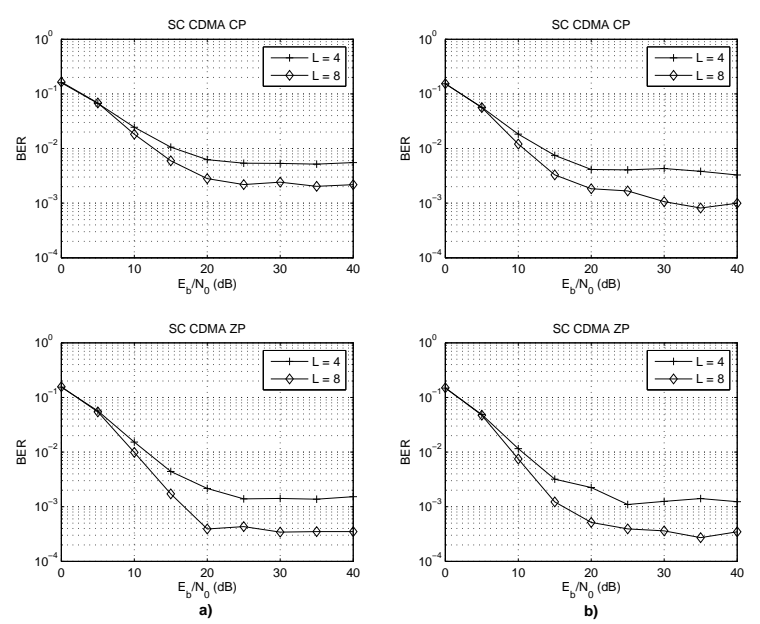

Fig. 3. BER versus $E_{b} / N_{0}$ for BPSK constellation with different channel orders, DD-MMSE receiver. a) PN b) Zadoff-Chu

proposed scheme outperforms the system in [18] for both SC CDMA CP case and SC CDMA ZP case. The use of ZadoffChu codes results in better performance in all cases.

\section{CONCLUSION}

In this work a new space-time scheme for single carrier CDMA block transmission was proposed. The proposed scheme uses Alamouti's space-time codes, and allow us to exploit its orthogonal property and to decode each transmit symbol separately. It was analysed the diversity gain of the systems and established conditions to achieve the maximum diversity gain, i.e., the conditions to fully exploit the multiantenna and multipath diversity.

It was proposed the decision directed minimum squared error receiver, which can use, depending on the user codes, multichannel and multipath components of the signal to enhance the bit error rate. It was shown that the proposed systems outperform previously proposed systems.
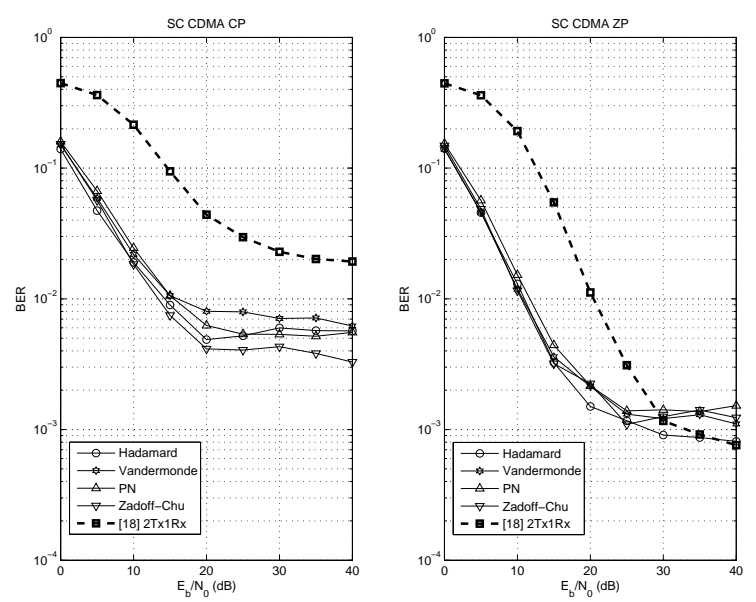

Fig. 4. BER versus $E_{b} / N_{0}$ for BPSK constellation and different codes, DD-MMSE receiver, $L=4$

\section{REFERENCES}

[1] M. Steer, "Beyond 3G," IEEE Microw. Mag., vol. 8, no. 1, pp. 76-82, February 2007.

[2] K. L. Baum, T. A. Thomas, F. W. Vook, and V. Nangia, "Cyclic-prefix CDMA: an improved transmission method for broadband DS-CDMA cellular systems," Proc. WCNC, vol. 1, pp. 183-188, March 2002.

[3] A. S. Madhukumar, F. Chin, Y-C. Liang, and K. Yang, "Single-carrier cyclic prefix-assisted CDMA system with frequency domain equalization for high data rate transmission," EURASIP J. Wirel. Commun. Netw., vol. 2004, no. 1, pp. 149-160, 2004.

[4] S. Barbarossa, Multiantenna Wireless Communication Systems, Artech House, 2005.

[5] S. Alamouti, "A simple transmit diversity technique for wireless communications," IEEE J. Sel. Areas Commun., vol. 16, no. 8, October 1998.

[6] V. Tarokh, H. Jafarkhani, and A. R. Calderbank, "Space-time block codes from orthogonal designs," IEEE Trans. Inf. Theory, vol. 45, no. 5 , pp. 1456-1467, July 1999.

[7] Z. Wang and G. B. Giannakis, "Wireless multicarrier communications: Where Fourier meets Shannon," IEEE Signal Process. Mag., pp. 29-47, May 2000.

[8] S. Zhou and G. Giannakis, "Single-carrier space-time block-coded transmission over frequency-selective fading channels," IEEE Trans. Inf. Theory, vol. 49, no. 1, pp. 164-179, January 2003.

[9] S. Zhou and G. Giannakis, "Space-time coding with maximum diversity gains over frequency-selective fading channels," IEEE Signal Process. Lett., vol. 48, no. 10, pp. 269-272, October 2001.

[10] Y. Xin, Z. Wang, and G. B. Giannakis, "Space-time diversity systems based on linear constellation precoding," IEEE Trans. Wireless Commun., vol. 2, no. 2, pp. 294-309, March 2003.

[11] A. S. Cacciapuoti, G. Gelli, and F. Verde, "FIR zero-forcing multiuser detection and code designs for downlink MC-CDMA," IEEE Trans. Signal Process., vol. 55, no. 10, pp. 4737-4751, October 2007.

[12] S. Haykin, Adaptive Filter Theory, Prentice Hall, 2001.

[13] L. S. Resende, J. M. Romano, and M. Bellanger, "A fast least-squares algorithm for linearly constrained adaptive filtering," IEEE Trans. Signal Process., vol. 44, no. 5, pp. 1168-1174, May 1996.

[14] R. L. Frank and S. A. Zadoff, "Phase shift pulse codes with good periodic correlation properties," IEEE Trans. Inf. Theory, vol. IT-8, pp. 381-382, October 1962.

[15] D. Chu, "Polyphase codes with good periodic correlation properties," IEEE Trans. Inf. Theory, vol. 18, no. 4, pp. 531-532, July 1972.

[16] T. S. Rappaport, Wireless Communications: Principles and Practice, Prentice Hall PTR, 1996.

[17] H. Li, X. Lu, and G. B. Giannakis, "Capon multiuser receiver for CDMA systems with space-time coding," IEEE Trans. Signal Process., vol. 50, no. 5, pp. 1193-1204, May 2002.

[18] C. A. Medina, T. T. V. Vinhoza, and R. Sampaio-Neto, "Performance comparison of space-time block coding for single and multicarrier CDMA systems," XXV Simpósio Brasileiro de Telecomunicações (SBrT 2007), Recife, Brazil, September 2007. 